Article

\title{
The Core of Gut Life: Firmicutes Profile in Patients with Relapsing-Remitting Multiple Sclerosis
}

\author{
Madina Kozhieva ${ }^{1}$, Natalia Naumova ${ }^{2, *}$ (D) Tatiana Alikina ${ }^{2}$, Alexey Boyko ${ }^{3}$, Valentin Vlassov ${ }^{2}$ and \\ Marsel R. Kabilov ${ }^{2}$ (D) \\ 1 Department of Neurology, Neurosurgery and Medical Genetics of the Pirogov Medical University, \\ 117513 Moscow, Russia; kozhieva.m@fccps.ru \\ 2 Institute of Chemical Biology and Fundamental Medicine SB RAS, 630090 Novosibirsk, Russia; \\ alikina@niboch.nsc.ru (T.A.); Valentin.Vlassov@niboch.nsc.ru (V.V.); kabilov@niboch.nsc.ru (M.R.K.) \\ 3 Department of Neuroimmunology of the Federal Center of CVPI, 117513 Moscow, Russia; boiko.a@fccps.ru \\ * Correspondence: naumova@niboch.nsc.ru or nnaumova@mail.ru
}

Citation: Kozhieva, M.; Naumova, N.; Alikina, T.; Boyko, A.; Vlassov, V.; Kabilov, M.R. The Core of Gut Life: Firmicutes Profile in Patients with Relapsing-Remitting Multiple Sclerosis. Life 2021, 11, 55. https:// doi.org/10.3390/life11010055

Received: 29 November 2020

Accepted: 11 January 2021

Published: 14 January 2021

Publisher's Note: MDPI stays neutral with regard to jurisdictional clai$\mathrm{ms}$ in published maps and institutional affiliations.

Copyright: (C) 2021 by the authors. Licensee MDPI, Basel, Switzerland. This article is an open access article distributed under the terms and conditions of the Creative Commons Attribution (CC BY) license (https:// creativecommons.org/licenses/by/ $4.0 /)$.
Abstract: The multiple sclerosis (MS) incidence rate has been increasing in Russia, but the information about the gut bacteriobiome in the MS-afflicted patients is scarce. Using the Illumina MiSeq sequencing of 16S rRNA gene amplicons, we aimed to analyze the Firmicutes phylum and its taxa in a cohort of Moscow patients with relapsing-remitting MS, assessing the effects of age, BMI, disease modifying therapy (DMT), disability (EDSS), and gender. Among 1252 identified bacterial OTUs, 857 represented Firmicutes. The phylum was the most abundant also in sequence reads, overall averaging $74 \pm 13 \%$. The general linear model (GLM) analysis implicated Firmicutes/Clostridia/Clostridiales/Lachospiraceae/Blautia/Blautia wexlerae as increasing with BMI, and only Lachospiraceae/Blautia/Blautia wexlerae as increasing with age. A marked DMT-related decrease in Firmicutes was observed in females at the phylum, class (Clostridia), and order (Clostridiales) levels. The results of our study implicate DMT and gender as factors shaping the fecal Firmicutes assemblages. Together with the gender-dependent differential MS incidence growth rate in the country, the results suggest the likely involvement of gender-specific pathoecological mechanisms underlying the occurrence of the disease, switching between its phenotypes and response to disease-modifying therapies. Overall, the presented profile of Firmicutes can be used as a reference for more detailed research aimed at elucidating the contribution of this core phylum and its lower taxa into the etiology and progression of relapsing-remitting multiple sclerosis.

Keywords: multiple sclerosis; relapsing-remitting course; fecal bacteriobiome; 16S rRNA gene amplicon sequencing

\section{Introduction}

The culture-independent identification of a plethora of gut microorganisms, which has been made possible by rapid advancements in methodology and instruments, has contributed to revealing the intimate relationship between the microbiota and the host, and by now there is no doubt that the gut microbiota significantly shapes human health [1-3]. Multiple sclerosis (MS) is one of the neurological disorders with increasing evidence implicating the gut microbiome as a key susceptibility factor [4-6]: MS patients have dysbiosis compared to healthy individuals [7-9], although the cause-effect relationship between gut microbiota dysbiosis and MS so far has not been unambiguously revealed [2,10,11]. Microbial involvement has been suggested as a cause of some chronic inflammatory diseases, including MS [12-14], and hence as a potential target for safer novel therapeutic strategies to treat the disease $[7,15]$. In the case of relapsing-remitting MS (RRMS), switching from relapse into a more prolonged remittance stage is a minimum. Such microbiota-based strategies need a better outline of the relationship between the microbiota and the host [16] in the RRMS-afflicted subjects in different regions of the world, elucidating the global picture. 
The pathological and clinical symptoms of MS may vary widely [17], often presenting a challenge for correct diagnostics [18,19]. Presently, the relapsing-remitting course of the disease is the most common [5,20] and, consequently, the most studied [5,21]. The differences existing between the microbiomes of MS patients and healthy subjects were found to be exacerbated in chloroform-resistant, spore-forming bacteria, which primarily belong to the phylum Firmicutes (the Bacilli and Clostridia classes) [22], the core phylum in the human gut [23-25].

However, some studies included fecal samples from RRMS patients only in the remission phase $[26,27]$. The geographic origin of the surveyed population may have a greater impact on the composition of the gut microbiota than BMI or sex $[23,28]$. The MS prevalence and incidence rate has been steadily increasing in Russia [29]. Based on all these factors, we decided to aim our study at obtaining a picture of the gut 16S rRNA gene amplicon sequence abundance and the diversity of the Firmicutes phylum and its taxa in a cohort of Russian RRMS patients in remission or relapse, and at assessing the effects of body mass index, disease modifying therapy, disability assessment, and sex within the cohort.

\section{Materials and Methods}

\subsection{Participants and Fecal Sample Collection}

Sixty-four patients with remitting-relapsing MS course, as diagnosed by the MacDonald criteria [30], which have been validated in Russia [31], were recruited for the trial (Table 1). All the patients underwent clinical examination to assess their neurological status and disability according to the Expanded Disability Status Scale (EDSS) [32]. Some of the patients received disease-modifying therapy (DMT) as high-dose interferon-beta- 1 . No other medicines were administered, as they could have modified the gut microbiome [33]. The patients had no history of treatment with antibiotics at least for 3 months prior to feces sampling, as well as no probiotics and/or probiotics as special supplementation. All the patients were duly informed, gave their consent to the study, and signed the informed consent.

Table 1. Demographics of the study cohort $(\mathrm{N}=64)$.

\begin{tabular}{ccccc}
\hline & Mean & Median & Min & Max \\
\hline Age, years & 37.8 & 37.0 & 19.0 & 62.0 \\
BMI $^{\$}, \mathrm{~kg} / \mathrm{m}^{2}$ & 24.0 & 23.4 & 18.3 & 35.6 \\
EDSS ${ }^{2}$ & 2.2 & 2.0 & 1.0 & 4.5 \\
\hline
\end{tabular}

$\$$ BMI stands for body mass index; ${ }^{\&}$ EDSS stands for expanded disability status scale.

Fecal samples were collected in $10 \mathrm{~mL}$ sterile fecal specimen containers and stored frozen at approximately $-20^{\circ} \mathrm{C}$. Samples were transferred to the laboratory within 1 week of collection and stored at $-80{ }^{\circ} \mathrm{C}$ until they could be used for DNA extraction. The samples were collected at least one month prior to the corticosteroid treatment.

The protocol of the study was approved by the Ethics Committee of the Pirogov National Science and Research Medical University. All the clinical aspects of the study were supervised by a neurologist. New medicines, sorbents, and/or laxatives (including magnesium salts and castor oil), as well as any diet changes, were cancelled or not started at least one week prior to the fecal sample collection.

\subsection{DNA Extraction and Sequencing}

DNA was extracted from 50 to $100 \mathrm{mg}$ of thawed patient fecal samples using the MetaHIT protocol [34]. The bead-beating was performed using TissueLyser II (Qiagen, Hilden, Germany) for $10 \mathrm{~min}$ at $30 \mathrm{~Hz}$. No further purification of the DNA was needed. The quality of the DNA was assessed using agarose gel electrophoresis.

The 16S rRNA gene region was amplified with the primer pair V3-V4 combined with Illumina adapter sequences [35]. PCR amplification was performed as described earlier [36]. All the PCR reactions used $25 \mathrm{ng}$ of fecal DNA as a template and were 
performed in triplicate. A total of $200 \mathrm{ng}$ PCR product from each sample was pooled together and purified through MinElute Gel Extraction Kit (Qiagen, Hilden, Germany). The obtained libraries were sequenced with $2 \times 300$ bp paired-ends reagents on MiSeq (Illumina, San Diego, CA, USA) in SB RAS Genomics Core Facility (ICBFM SB RAS, Novosibirsk, Russia). The read data were deposited in GenBank under the study accession number PRJNA680445.

\subsection{Bioinformatic and Statistical Analyses}

Raw sequences were analyzed with the UPARSE pipeline [37] using Usearch v10.0. The UPARSE pipeline included the merging of paired reads; read quality filtering; length trimming; the merging of identical reads (dereplication); discarding singleton reads; removing chimeras; and operational taxonomic unit (OTU) clustering using the UPARSE-OTU algorithm. The OTU sequences were assigned a taxonomy using the SINTAX [38] and 16S RDP training set v16 [39].

The taxonomic structure of the obtained sequence assemblages-i.e., a collection of different species at one site at one time [40] — was estimated by the ratio of the number of taxon-specific sequence reads to the total number of sequence reads-i.e., by the relative abundance of taxa-which was expressed as a percentage.

The results are expressed as a mean and standard deviation (s.d.). Normal distribution was assessed using the Shapiro-Wilk test. A general linear model with two categorical factors (DMT and sex) and three continuous factors (age, BMI, and EDSS) was used to assess the influence of demographic and therapeutic variables on the Firmicutes abundance. Between-group comparisons were carried out as a post hoc analysis using the Fisher's LSD test in the Statistica v.13.3 software (Statsoft, Tulsa, OK, USA). Alpha-biodiversity indices were calculated using Usearch.

\section{Results}

\subsection{Total Bacteriobiome Diversity}

After the quality filtering and chimera removal, a total of 1256 different OTUs were identified at a $97 \%$ sequence identity level, of which the overwhelming majority (1252) was Bacteria, with the other four representing the Euryarchaeota phylum of the Archaea domain.

Twelve bacterial phyla were identified, containing 21 classes, 25 orders, 54 families, and 174 genera, along with unidentified taxa. Most of the bacterial OTUs represented the Firmicutes phylum (857 OTUs, or ca. $68 \%$ of the total bacterial OTU number), with Bacteroidetes and Actinobacteria being the second and the third most OTU-rich phyla, with 148 $(12 \%)$ and 84 OTUs $(7 \%)$, respectively. Clostridia was the OTU-richest class (669 OTUs), accounting for $53 \%$ of the total OTU richness, with Bacteroidia (135 OTUs) and Actinobacteria (79) contributing $11 \%$ and $6 \%$, respectively. Thus, these three classes drastically prevailed in the fecal bacteriobiomes studied.

Overall in the samples, the number of dominant OTUs-i.e., OTUs contributing $\geq 1 \%$ of the total sequence number in a sample-was 29 , i.e., $2.0 \%$ of the total number of OTUs. They represented four phyla (Firmicutes, Bacteroidetes, Actinobacteria, and Proteobacteria), six classes (Clostridia, Bacilli, Negativicutes, Bacteroidia, Actinobacteria, and Gammaproteobacteria), 8 orders, 11 families, and 20 genera-i.e., far less taxonomic richness as compared to the total list of identified OTUs.

Members of the Firmicutes phylum were by far the most abundant not only in OTU richness but also in the relative abundance of sequence reads, which averaged $74 \pm 13 \%$ for the studied cohort. The phylum was the only one with its relative abundance data complying with the normal distribution, as judged on the basis of Shapiro-Wilk's test and the normal probability plots. Within the phylum's lower taxonomic levels, Clostridia, Clostridiales, Ruminococcaceae, Fecalibacterium, and Fecalibacterium prausnitzii also showed a distribution pattern complying with the normal one. The performed statistical analysis, using GLM to elucidate the effect of age, body mass index, and EDSS as continuous factors (covariates) and sex and disease-modifying therapy as categorical factors, did not show age, 
BMI, and EDSS effects on Ruminococcaceae (Tables S4, S6 and S8) and its lower taxa, whereas the taxa Lachospiraceae/Blautia/Blautia wexlerae (Table 2; Tables S5, S7 and S9) had some ageand BMI-related shifts in their relative abundance. It should be noted that although the data on the relative abundance of Lachospiraceae and its Blautia genus and Blautia wexlerae were not normally distributed, their residuals were, therefore the GLM results pertaining to the taxa were taken into consideration. The profiles for the GLM-predicted relative abundance visualize these effects (Figure 1). The BMI effect in the studied cohort was revealed already at the phylum level (the predicted relative abundance is shown in Figure 1a), and further down at the class and order levels. The effect of sex and EDSS as individual factors was not statistically significant, with both making negligible contributions to the total data variance (Table 2).

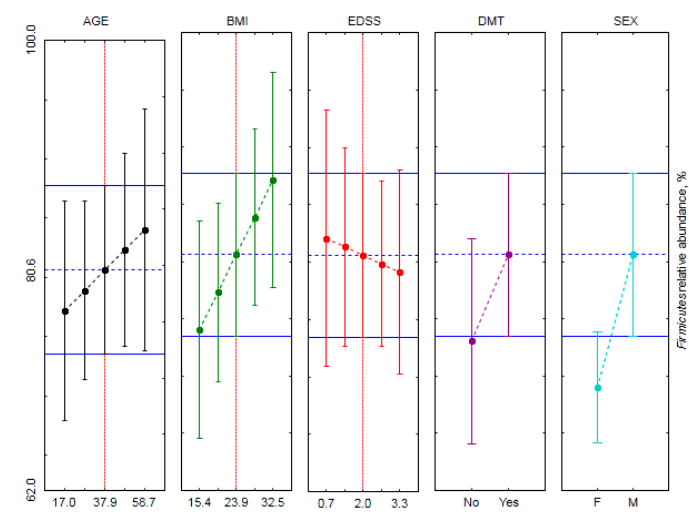

(a)

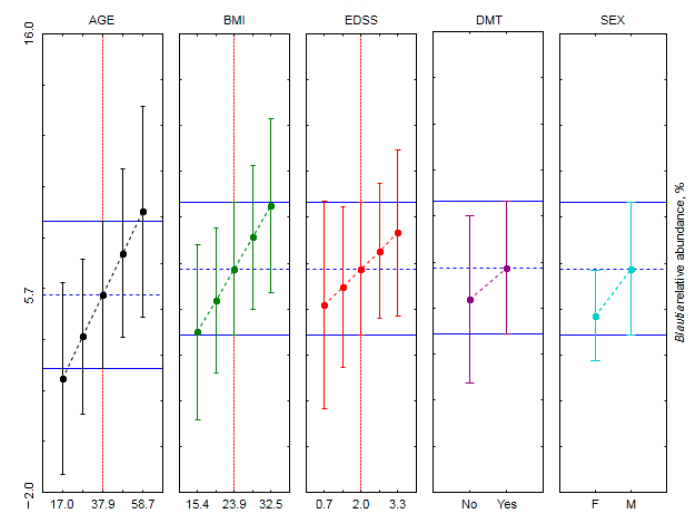

(c)

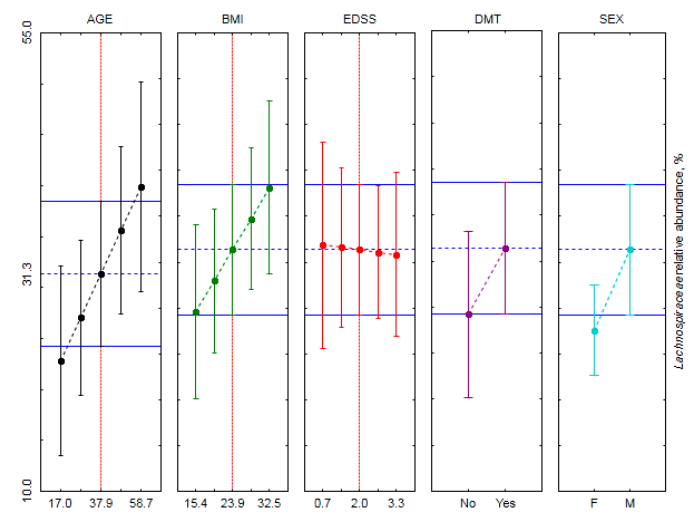

(b)

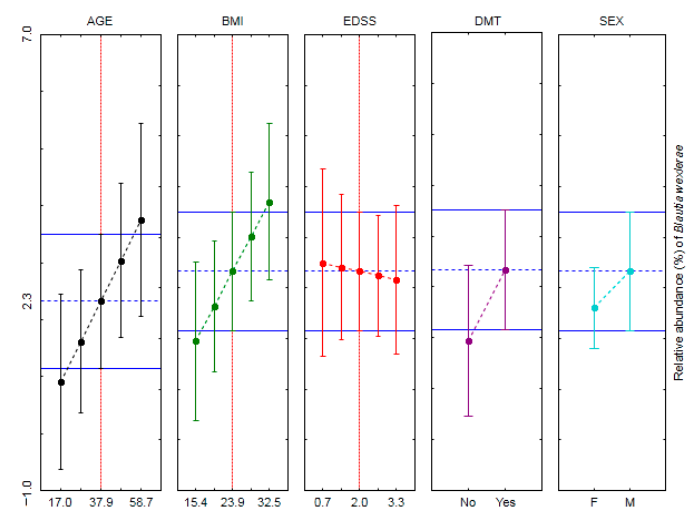

(d)

Figure 1. Profiles for the GLM-predicted values of relative abundance: (a) Firmicutes, (b) Lachnospiraceae, (c) Blautia, and (d) Blautia wexlerae. Markers show mean values and whiskers show 0.95 confidence intervals. DMT: No-no therapy. Yes-interferon treatment. Sex: $\mathrm{F}$-females, $\mathrm{M}$-males.

\subsection{DMT Effect on the Firmicutes Taxa Abundance}

Disease-modifying therapy as a separate factor was found to have a negligible effect, both microbially and statistically, on the Firmicutes taxa abundance (Table 2, Tables S1-S9). However, quite a noticeable portion of the data variance was accounted for by the interaction between DMT and the patients' sex (Table 2); a marked DMT-related decrease in Firmicutes was observed in females at the phylum, class, and order levels (Table 3, Tables S1-S3), and at the family level only for Lachnospiraceae (Table S5). At the same levels, there was a difference between females and males in the DMT subcohort, with males having substantially (more than 10\%) increased relative abundance at the phylum, class, and order levels (Table 3) and the effect decreasing in its size and statistical significance at the family, genus, and species levels (Lachnospiraceae/Blautia/Blautia wexlerae). 
Table 2. General linear model results for the factors' contribution to the variance in different taxa sequence reads' relative abundance.

\begin{tabular}{|c|c|c|c|c|c|c|c|c|c|}
\hline \multirow[b]{3}{*}{ Factor } & \multicolumn{9}{|c|}{ Taxonomic Level } \\
\hline & \multirow{2}{*}{$\begin{array}{c}\text { Phylum } \\
\text { Firmicutes }\end{array}$} & \multirow{2}{*}{$\begin{array}{c}\text { Class } \\
\text { Clostridia }\end{array}$} & \multirow{2}{*}{$\begin{array}{c}\text { Order } \\
\text { Clostridiales }\end{array}$} & \multicolumn{2}{|c|}{ Family } & \multicolumn{2}{|c|}{ Genus } & \multicolumn{2}{|l|}{ OTU } \\
\hline & & & & Ruminococcaceae & Lachnospiraceae & Fecalibacterium & Blautia & Fecalibacterium prausnitzii & Blautia wexlerae \\
\hline $\mathrm{BMI}^{\$}$ & 6.3 & 6.2 & 6.8 & 0.5 & 6.4 & 1.4 & 6.6 & 1.3 & 9.7 \\
\hline EDSS \& & 0.2 & 0.1 & 0.1 & 0.0 & 0.0 & 0.5 & 1.6 & 0.6 & 0.1 \\
\hline DMT \# & 0.0 & 0.4 & 0.5 & 0.6 & 0.3 & 0.1 & 0.5 & 0.1 & 0.5 \\
\hline Sex & 1.6 & 2.4 & 2.3 & 4.1 & 0.0 & 0.0 & 0.1 & 0.0 & 0.4 \\
\hline Error & 79.6 & 75.5 & 76.5 & 89.3 & 74.9 & 93.6 & $\overline{76.7}$ & 93.7 & $\overline{75.1}$ \\
\hline
\end{tabular}

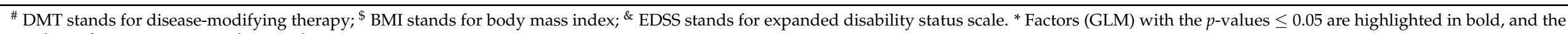
$p$-values of $0.05 \leq 0.10$ are underscored.

Table 3. Relative abundance (\%) of Firmicutes-specific sequence reads.

\begin{tabular}{|c|c|c|c|c|c|c|c|c|c|c|c|c|}
\hline & \multirow{3}{*}{\multicolumn{2}{|c|}{ Factor Rate }} & \multirow{3}{*}{$\mathbf{N}$} & \multicolumn{9}{|c|}{ Taxon Level } \\
\hline & & & & \multirow{2}{*}{\begin{tabular}{|c} 
Phylum \\
Firmicutes
\end{tabular}} & \multirow{2}{*}{$\begin{array}{c}\text { Class } \\
\text { Clostridia } \\
\end{array}$} & \multirow{2}{*}{$\begin{array}{c}\text { Order } \\
\text { Clostridiales }\end{array}$} & \multicolumn{2}{|c|}{ Family } & \multicolumn{2}{|l|}{ Genus } & \multicolumn{2}{|l|}{ Species } \\
\hline & & & & & & & Ruminococcaceae & Lachnospiraceae & Fecalibacterium & Blautia & Fecalibacterium prausnitzii & Blautia wexlerae \\
\hline Entire cohort & & & 64 & 74.0 & 66.0 & 65.0 & 31.0 & 27.3 & 11.0 & 5.1 & 10.9 & 1.8 \\
\hline Sex & $\begin{array}{c}\text { Females } \\
\text { Males }\end{array}$ & & $\begin{array}{l}44 \\
20 \\
\end{array}$ & $\begin{array}{l}72.4 \\
77.5 \\
\end{array}$ & $\begin{array}{l}63.8 \\
70.7 \\
\end{array}$ & $\begin{array}{l}62.8 \\
69.8 \\
\end{array}$ & $\begin{array}{l}29.5 \\
34.2 \\
\end{array}$ & $\begin{array}{l}26.9 \\
28.1\end{array}$ & $\begin{array}{l}10.8 \\
11.4 \\
\end{array}$ & $\begin{array}{l}5.1 \\
5.0\end{array}$ & $\begin{array}{l}10.7 \\
11.4 \\
\end{array}$ & $\begin{array}{l}1.9 \\
1.7 \\
\end{array}$ \\
\hline $\mathrm{DMT} \times$ Sex & $\begin{array}{c}\text { No } \\
\text { No } \\
\text { DMT } \\
\text { DMT }\end{array}$ & $\begin{array}{c}\text { Females } \\
\text { Males } \\
\text { Females } \\
\text { Males }\end{array}$ & $\begin{array}{c}17 \\
8 \\
27 \\
12\end{array}$ & $\begin{array}{c}77.0 \mathrm{~b}^{*} \\
73.8 \mathrm{ab} \\
69.4 \mathrm{a} \\
80.0 \mathrm{~b}\end{array}$ & $\begin{array}{c}70.1 \mathrm{~b} \\
67.0 \mathrm{ab} \\
59.8 \mathrm{a} \\
73.2 \mathrm{~b}\end{array}$ & $\begin{array}{c}69.1 \mathrm{~b} \\
66.5 \mathrm{ab} \\
58.9 \mathrm{a} \\
72.0 \mathrm{~b}\end{array}$ & $\begin{array}{l}32.0 \\
34.8 \\
27.9 \\
33.7\end{array}$ & $\begin{array}{l}\frac{31.3 \mathrm{~b}}{24.2 \mathrm{ab}} \\
\underline{24.1 \mathrm{a}} \\
\underline{30.7 \mathrm{ab}} \\
\end{array}$ & $\begin{array}{c}12.6 \\
11.0 \\
9.7 \\
11.7\end{array}$ & $\begin{array}{l}5.8 \\
3.6 \\
4.6 \\
5.9\end{array}$ & $\begin{array}{c}12.5 \\
11.0 \\
9.7 \\
11.6\end{array}$ & $\begin{array}{l}1.9 \\
1.0 \\
1.8 \\
2.2\end{array}$ \\
\hline
\end{tabular}

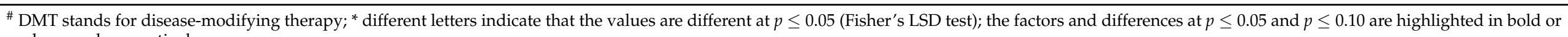
underscored, respectively. 
The $\alpha$-biodiversity indices calculated only for the Firmicutes OTUs were also subjected to GLM analysis and showed no DMT-related differential abundance (Table 4), averaging for the entire cohort $243 \pm 56$ observed OTUs (species richness), $299 \pm 70$ all OTUs (Chao-1), $3.51 \pm 0.03$ (Shannon), $0.145 \pm 0.030$ (evenness), and Fisher's $\alpha(39 \pm 10)$. However, the interaction between DMT and sex had an effect on the evenness, as DMT decreased this $\alpha$-biodiversity index by $15 \%$ in males.

Table 4. Alpha-biodiversity indices for Firmicutes OTUs in the fecal samples of patients with relapsing-remitting multiple sclerosis.

\begin{tabular}{|c|c|c|c|c|c|c|c|c|}
\hline & \multicolumn{2}{|c|}{ Factor Rate } & $\mathbf{N}$ & Richness (OTUs) & Chao-1 & Shannon & Evenness & Fisher's $\alpha$ \\
\hline Entire cohort & & & 64 & 243 & 299 & 3.51 & 0.145 & 39 \\
\hline \multirow{2}{*}{$\mathrm{DMT}^{\#}$} & No & & 25 & 243 & 299 & 3.47 & 0.140 & 39 \\
\hline & DMT & & 39 & 243 & 299 & 3.54 & 0.149 & 39 \\
\hline \multirow{2}{*}{ Sex } & Females & & 44 & 246 & 303 & 3.56 & 0.150 & 40 \\
\hline & Males & & 20 & 237 & 290 & 3.42 & 0.134 & 38 \\
\hline \multirow{4}{*}{ DMT $\times$ Sex } & No & Females & 17 & 246 & 303 & 3.49 & $0.141 a^{*}$ & 40 \\
\hline & No & Males & 8 & 247 & 303 & 3.60 & $0.156 b$ & 40 \\
\hline & DMT & Females & 27 & 239 & 291 & 3.44 & $0.137 \mathrm{ab}$ & 38 \\
\hline & DMT & Males & 12 & 236 & 289 & 3.41 & 0.133 a & 38 \\
\hline
\end{tabular}

" DMT stands for disease modifying therapy; * different letters indicate that the values are different at $p \leq 0.05$ (Fisher's LSD test); the factors and differences at $p \leq 0.05$ are highlighted in bold.

\section{Discussion}

Our study of the cohort of patients with relapsing-remitting MS found Firmicutes to be ultimately prevailing in the fecal bacteriobiome, accounting for more than three quarters of the relative abundance. In general, the finding agrees with the established global pattern of the phylum prevalence, albeit with some exceptions [41], in the gut/fecal bacteriobiome of both healthy individuals $[24,42-44]$ and those compromised by various disorders/diseases $[41,45,46]$. In this study, we did not aim at comparing the RRMS cohort with healthy individuals, yet a lower percentage of Firmicutes was often reported for healthy cohorts elsewhere [26,42,47-50]. As for comparing our RRMS cohort with the RRMS cohorts of other ethnicity/regions, Firmucutes was at least $20 \%$ more abundant in the studied cohort as compared with the Italian and Japanese ones [26,51]; the effect very likely resulted from the stark national differences in diet between these cohorts.

The gut microbiota diversity is generally believed to change with age [44,52], and our cohort was not an exception, as age accounted for several percent of the variance in the Lachnospiraceae family, the second-ranked family in terms of relative abundance in the Firmicutes phylum; its Blautia genus; and Blautia wexlerae, one of the main dominant species in the study, with a ca. $2 \%$ abundance. Recently, a higher relative abundance of Blautia was reported in healthy Dutch adults as compared with children [53], and in children with cystic fibrosis Blautia was reported to be positively associated with age, the latter being the strongest predictor of overall fecal bacterial diversity [54]. However, we could not find reported association specifically between Blautia/Blautia wexlerae with the age of the MS-afflicted patients.

The Firmicutes abundance was reported to be associated with BMI in healthy individuals $[55,56]$. However, the information is sometimes confusing, as some studies reported increased BMI-related abundance [50,57], whereas others reported a decreased one [58]. As for the Firmicutes lower taxa, Blautia, for instance, was shown to be significantly and inversely associated with visceral fat accumulation by healthy Japanese people of both sexes [42]. Therefore, our finding that BMI accounts for several percent of the Firmicutes taxa abundance variance, with Blautia/Blautia wexlerae being mostly responsible for the effect (increase), apparently either complies or contradicts other reported data, in any case 
contributing to the general outline of RRMS bacteriobiome. Additionally, we could not find the information about Blautia abundance associated with BMI in other RRMS cohorts to make any comparison, so the implications of such association for disease phenotype and overall progression remains to be determined.

It is difficult to interpret changes in the Blautia/Blautia wexlerae presence in fecal assemblages in terms of the benefit for human health, as the reported data can be confusing or lacking. The overabundance of Blautia was reported in a Russian cohort with prediabetes and type 2 diabetes [57]; however, the genus was ultimately dominant also in healthy subjects with a normal level of glucose tolerance. On the other hand, some studies report Blautia as being proinflammatory [58].

Our finding that sex as a separate factor had not contributed to the data variance of the Firmicutes gut fecal assemblages of the RRMS patients may indicate the effect of some equalizing (in this respect) factor, since sex-related differences in the gut microbiota were revealed in some studies with autoimmune diseases [59].

Our result that disease-modifying therapy as an individual factor had not significantly contributed to the data variance was unexpected, as previous studies revealed DMT-related differences in the gut microbiota of MS patients: the dimethyl fumarate or interferon $\beta$-b treatment decreased the Firmicutes phylum relative abundance $[60,61]$. Yet, other studies reported an increased Firmicutes abundance, mostly driven by Fecalibacterium, in the dimethyl fumarate-treated patients [62], or no difference in the Firmicutes abundance between no-DMT and DMT cohorts [61]. However, in our study the observed 2\% decrease in the Fecalibacterium abundance was not statistically significant and could hardly be physiologically relevant with its negligible contribution to the data variance. Our finding of sex-related differences in Firmicutes abundance between the no-DMT and DMT-subcohorts strongly suggests that there exists a sex-dependent effect on modulating the gut Firmicutes abundance according to DMT, at least in the studied RRMS cohort. The effect may be partially due to sex-associated diet differences $[51,57,63,64]$, as the studied RRMS patients did not follow any specific diet recommendations.

The studied cohort included RRMS patients with clinical and neurodegenerative evidence of the two phenotypes of the disease activity-i.e., remission or relapse. However, the performed GLM analysis using EDSS as continuous factor, rather than using phenotype as a categorical one, showed that in the studied RRMS cohort there was no association between EDSS and the Firmicutes phylum and its lower taxa abundance. This is apparently in contrast with other studies that reported Firmicutes to be increased in MS patients during times of higher disease activity, and decreased at disease quiescence [26]. However, the discrepancy is very likely due to the absence of total synonymy between the EDSS and the clinical assessment of the disease activity phenotype, as well as other confounding factors-e.g., the timing and duration of DMT, etc.

Fecalibacterium representatives, and specifically Fecalibacterium prausnitzii, are known to metabolize dietary fibers as major short-chain fatty acid producers providing energy sources for enterocytes and achieving anti-inflammatory effects in the gut [65], and hence are generally considered to be beneficial for human health. The studied RRMS cohort overall had $11 \%$ of the genus relative abundance, thus being very close to the RRMS cohorts of some other ethnicities/races [66]. However, as the genus abundance showed no association with EDSS or DMT, it does not seem to be a promising target for microbiota modulation therapies to benefit RRMS patients' condition.

Finally, we would reiterate some aspects pertaining to interpreting the fecal microbiome data in disease. Firstly, the overwhelming majority of the published results, based on the data for stool/feces samples, refer to such results euphemistically as the "gut microbiome" or the "intestinal microbiome", rather than the "fecal microbiome". However, as S. Fujio-Vejar et al. (2017) [42] rightly noted, the human fecal microbiota is not an entirely reliable reflection of the cecal or colonic microbiota. Secondly, a high inter-individual variability is observed in the studied populations/cohorts/groups, and our study is not an exception. The heterogeneity is usually attributed to an unspecified and hence unaccounted 
for plethora of influencing factors. However, one feces sample is usually collected from a sole stool event-i.e., without any individual replication. The latter, if performed, will decrease the intra-individual variability, consequently reducing some of the inter-individual one. Thirdly, we cannot help but note that associations between fecal/gut microbiota composition and host characteristics neither suggest nor prove any cause-effect dependence, allowing for two-way interpretation; the increased abundance of a certain taxon, especially apparently non-beneficial microbes, may be the essential enhancing agent of a disease, but it can also be one of the host's means of adapting and/or mitigating some pathoecophysiological processes, triggered in a host by genetic and/or external factors. Last but not least, one should always bear in mind that the proportions of gene copy numbers, be they $16 \mathrm{~S}$ rRNA or functional genes, are not entirely synonymic with the number of the relevant organisms present and the intensity of the processes they perform. Thus, the microbiome profiles provide just scaffolding that will be useful for constructing more comprehensive and/or targeted research.

\section{Conclusions}

Here, we presented the first gut bacteriobiome profiling of a Russian cohort of patients with relapsing-remitting multiple sclerosis, focusing solely on the Firmicutes phylum as the ultimate dominant and its taxa abundance as related to age, sex, body mass index, disease-modifying therapy, and disability assessment. The results of our study implicate sex as a modulating/driving factor in the fecal Firmicutes assemblages examined. In general, the results comply with the sex-dependent differential incidence growth rate in Russia, which suggests sex-specific peculiarities in the pathophysiology of the disease. Our results also suggest the likely involvement of sex-specific pathoecological mechanisms underlying the occurrence and spread of the disease, switching between its phenotypes and response to disease-modifying therapies. We believe that our findings make a small but important contribution to constructing a global picture of the intestinal microbiota diversity in relapsing-remitting MS patients. The presented profile of Firmicutes indicates the avenues for detailed microbiological and physiological research aimed at elucidating the contribution of this core phylum and its taxa into the etiology and progression of relapsing-remitting multiple sclerosis.

Supplementary Materials: The following are available online at https:/ /www.mdpi.com/2075-172 9/11/1/55/s1: Table S1. GLM results for the Firmicutes-specific sequence reads abundance. Table S2. GLM results for the Clostridia-specific sequence reads abundance. Table S3. GLM results for the Clostridiales-specific sequence reads abundance. Table S4. GLM results for the Ruminococcaceaespecific sequence reads abundance. Table S5. GLM results for the Lachnospiraceae-specific sequence reads abundance. Table S6. GLM results for the Faecalibacterium-specific sequence reads abundance. Table S7. GLM results for the Blautia-specific sequence reads abundance. Table S8. GLM results for the Faecalibacterium prausnitzii-specific sequence reads abundance. Table S9. GLM results for the Blautia wexlerae specific sequence reads abundance.

Author Contributions: Conceptualization, A.B. and V.V.; methodology, M.K. and M.R.K.; software, M.R.K.; validation, N.N., T.A. and M.R.K.; formal analysis, N.N.; investigation, M.K. and T.A.; resources, M.R.K. and V.V.; data curation, M.K.; writing—original draft preparation, N.N.; writingreview and editing, A.B., V.V. and M.R.K.; visualization, N.N.; supervision, V.V.; project administration, A.B.; funding acquisition, V.V. All the authors have read and agreed to the published version of the manuscript.

Funding: This research was funded by the RUSSIAN FOUNDATION FOR BASIC RESEARCH, research project number 17-00-00295 (17-00-00210 and 17-00-00206). Bioinformatic analysis was partially supported by the MINISTRY OF SCIENCE AND HIGHER EDUCATION OF THE RUSSIAN FEDERATION, project number AAAA-A17-117020210021-7.

Institutional Review Board Statement: The the study was approved by the Ethic Committee of the PIROGOV NATIONAL SCIENCE AND RESEARCH MEDICAL UNIVERSITY (Moscow, Russia; (protocol No. 107 dated 25/10/2016). 
Informed Consent Statement: All patients were duly informed and gave their consent to the study and signed the Information Consent.

Data Availability Statement: The read data were deposited in GenBank under the study accession number PRJNA680445, and are available at https:/ / www.ncbi.nlm.nih.gov/bioproject/PRJNA680445/.

Conflicts of Interest: The authors declare no conflict of interest.

\section{References}

1. Opazo, M.C.; Ortega-Rocha, E.M.; Coronado-Arrázola, I.; Bonifaz, L.C.; Boudin, H.; Neunlist, M.; Riedel, C.A. Intestinal Microbiota Influences Non-intestinal Related Autoimmune Diseases. Front. Microbiol. 2018, 9, 432. [CrossRef] [PubMed]

2. Li, B.; Selmi, C.; Tang, R.; Gershwin, M.E.; Ma, X. The microbiome and autoimmunity: A paradigm from the gut-liver axis. Cell Mol. Immunol. 2018, 15, 595-609. [CrossRef] [PubMed]

3. Ogunrinola, G.A.; Oyewale, J.O.; Oshamika, O.O.; Olasehinde, G.I. The Human Microbiome and Its Impacts on Health. Int. J. Microbiol. 2020, 8045646. [CrossRef] [PubMed]

4. Cryan, J.F.; O’Riordan, K.J.; Sandhu, K.; Peterson, V.; Dinan, T.G. The gut microbiome in neurological disorders. Lancet Neurol. 2020, 19, 179-194. [CrossRef]

5. Mirza, A.; Forbes, J.D.; Zhu, F.; Bernstein, C.N.; van Domselaar, G.; Graham, M.; Waubant, E.; Tremlett, H. The multiple sclerosis gut microbiota: A systematic review. Mult. Scler. Relat. Disord. 2020, 37, 101427. [CrossRef]

6. Zhu, F.; Ju, Y.; Wang, W.; Wang, Q.; Guo, R.; Ma, Q.; Sun, Q.; Fan, Y.; Xie, Y.; Yang, Z.; et al. Metagenome-wide association of gut microbiome features for schizophrenia. Nat. Commun. 2020, 11, 1612. [CrossRef]

7. Chen, J.; Chia, N.; Kalari, K.; Yao, J.Z.; Novotna, M.; Paz Soldan, M.M.; Luckey, D.H.; Marietta, E.V.; Jeraldo, P.R.; Chen, X.; et al. Multiple sclerosis patients have a distinct gut microbiota compared to healthy controls. Sci. Rep. 2016, 6, 28484. [CrossRef]

8. Kirby, T.O.; Ochoa-Repáraz, J. The Gut Microbiome in Multiple Sclerosis: A Potential Therapeutic Avenue. Med. Sci. 2018, 6, 69. [CrossRef]

9. Wing, A.C.; Kremenchutzky, M. Multiple sclerosis and fecal microbiome transplantation: Are you going to eat that? Benef. Microbes. 2019, 10, 27-32. [CrossRef]

10. Bell, J.S.; Spencer, J.I.; Yates, R.L.; Yee, S.A.; Jacobs, B.M.; DeLuca, G.C. Invited Review: From nose to gut-The role of the microbiome in neurological disease. Neuropathol. Appl. Neurobiol. 2019, 45, 195-215. [CrossRef]

11. Boziki, M.K.; Kesidou, E.; Theotokis, P.; Mentis, A.A.; Karafoulidou, E.; Melnikov, M.; Sviridova, A.; Rogovski, V.; Boyko, A.; Grigoriadis, N. Microbiome in Multiple Sclerosis; Where Are We, What We Know and Do Not Know. Brain Sci. 2020, 10, 234. [CrossRef]

12. Kell, D.B.; Pretorius, E. No effects without causes: The Iron Dysregulation and Dormant Microbes hypothesis for chronic, inflammatory diseases. Biol. Rev. 2018, 93, 1518-1557. [CrossRef] [PubMed]

13. Sato, W.; Yamamura, T. Multiple sclerosis: Possibility of a gut environment-induced disease. Neurochem. Int. 2019, $130,104475$. [CrossRef] [PubMed]

14. Toghi, M.; Bitarafan, S.; Kasmaei, H.D.; Ghafouri-Fard, S. Bifidobacteria: A probable missing puzzle piece in the pathogenesis of multiple sclerosis. Mult. Scler. Relat. Disord. 2019, 36, 101378. [CrossRef] [PubMed]

15. Stanisavljević, S.; Dinić, M.; Jevtić, B.; Đedović, N.; Momčilović, M.; Đokić, J.; Golić, N.; Mostarica Stojković, M.; Miljković, Đ. Gut Microbiota Confers Resistance of Albino Oxford Rats to the Induction of Experimental Autoimmune Encephalomyelitis. Front. Immunol. 2018, 9, 942. [CrossRef] [PubMed]

16. Freedman, S.N.; Shahi, S.K.; Mangalam, A.K. The Gut Feeling: Breaking Down the Role of Gut Microbiome in Multiple Sclerosis. Neurotherapeutics 2018, 15, 109-125. [CrossRef] [PubMed]

17. Imitola, J. New age for progressive multiple sclerosis. Proc. Natl. Acad. Sci. USA 2019, 116, 8646-8648. [CrossRef]

18. Brownlee, W.J.; Hardy, T.A.; Fazekas, F.; Miller, D.H. Multiple Sclerosis. 1. Diagnosis of multiple sclerosis: Progress and challenges. Lancet 2016, 389, 1336-1346. [CrossRef]

19. Solomon, A.J.; Corboy, J.R. The tension between early diagnosis and misdiagnosis in multiple sclerosis. Nat. Rev. Neurol. 2017, 13, 567-572. [CrossRef]

20. Brenton, J.N.; Goldman, M.D. A study of dietary modification: Perceptions and attitudes of patients with multiple sclerosis. Mult. Scler. Relat. Disord. 2016, 8, 54-57. [CrossRef]

21. Gandy, K.A.O.; Zhang, J.; Nagarkatti, P.; Nagarkatti, M. The role of gut microbiota in shaping the relapse-remitting and chronic-progressive forms of multiple sclerosis in mouse models. Sci. Rep. 2019, 9, 6923. [CrossRef] [PubMed]

22. Cekanaviciute, E.; Pröbstel, A.-K.; Thomann, A.; Runia, T.F.; Casaccia, P.; Katz Sand, I.; Crabtree, E.; Singh, S.; Morrissey, J.; Barba, P.; et al. Multiple sclerosis-associated changes in the composition and immune functions of spore-forming Bacteria. mSystems 2018, 3, e00083-18. [CrossRef]

23. Escobar, J.S.; Klotz, B.; Valdes, B.E.; Agudelo, G.M. The gut microbiota of Colombians differs from that of Americans, Europeans and Asians. BMC Microbiol. 2014, 14, 311. [CrossRef] [PubMed]

24. Afolayan, A.O.; Ayeni, F.A.; Moissl-Eichinger, C.; Gorkiewicz, G.; Halwachs, B.; Högenauer, C. Impact of a Nomadic Pastoral Lifestyle on the Gut Microbiome in the Fulani Living in Nigeria. Front. Microbiol. 2019, 10, 2138. [CrossRef] [PubMed] 
25. Parbie, P.K.; Mizutani, T.; Ishizaka, A.; Kawana-Tachikawa, A.; Runtuwene, L.R.; Seki, S.; Abana, C.Z.; Kushitor, D.; Bonney, E.Y.; Ofori, S.B.; et al. Fecal microbiome composition in healthy adults in Ghana. JAP J. Inf. Dis. 2020. [CrossRef]

26. Cosorich, I.; Dalla-Costa, G.; Sorini, C.; Ferrarese, R.; Messina, M.J.; Dolpady, J.; Radice, E.; Mariani, A.; Testoni, P.A.; Canducci, F.; et al. High frequency of intestinal $\mathrm{T}(\mathrm{H}) 17$ cells correlates with microbiota alterations and disease activity in multiple sclerosis. Sci. Adv. 2017, 3, e1700492. [CrossRef]

27. Miyake, S.; Kim, S.; Suda, W.; Oshima, K.; Nakamura, M.; Matsuoka, T.; Chihara, N.; Tomita, A.; Sato, W.; Kim, S.W.; et al. Dysbiosis in the Gut Microbiota of Patients with Multiple Sclerosis, with a Striking Depletion of Species Belonging to Clostridia XIVa and IV Clusters. PLoS ONE 2015, 10, e0137429. [CrossRef] [PubMed]

28. Lan, D.; Ji, W.; Lin, B.; Chen, Y.; Huang, C.; Xiong, X.; Fu, M.; Mipam, T.D.; Ai, Y.; Zeng, B.; et al. Correlations between gut microbiota community structures of Tibetans and geography. Sci. Rep. 2017, 7, 16982. [CrossRef]

29. Boyko, A.N.; Melnikov, M. Prevalence and Incidence of Multiple Sclerosis in Russian Federation: 30 Years of Studies. Brain Sci. 2020, 10, 305. [CrossRef]

30. Polman, C.H.; Reingold, S.C.; Banwell, B.; Clanet, M.; Cohen, J.A.; Filippi, M.; Fujihara, K.; Havrdova, E.; Hutchinson, M.; Kappos, L.; et al. Diagnostic criteria for multiple sclerosis: 2010 revisions to the McDonald criteria. Ann. Neurol. 2011, 69, 292-302. [CrossRef]

31. Belova, A.N.; Shalenkov, I.V.; Shakurova, D.N.; Boyko, A.N. Revised McDonald criteria for multiple sclerosis diagnostics in central Russia: Sensitivity and specificity. Mult. Scler. J. 2014, 20, 1896-1899. [CrossRef] [PubMed]

32. Kurtzke, J.F. Rating neurologic impairment in multiple sclerosis. An Expanded disability status scale (EDSS). Neurology 1983, 39 , 291-302. [CrossRef] [PubMed]

33. Rumah, K.R.; Vartanian, T.K.; Fischetti, V.A. Oral Multiple Sclerosis Drugs Inhibit the In vitro Growth of Epsilon Toxin Producing Gut Bacterium, Clostridium perfringens. Front. Cell Infect. Microbiol. 2017, 7, 11. [CrossRef]

34. Godon, J.J.; Zumstein, E.; Dabert, P.; Habouzit, F.; Moletta, R. Molecular microbial diversity of an anaerobic digestor as determined by small-subunit rDNA sequence analysis. Appl. Environ. Microbiol. 1997, 63, 2802-2813. [CrossRef] [PubMed]

35. Fadrosh, D.W.; Ma, B.; Gajer, P.; Sengamalay, N.; Ott, S.; Brotman, R.M.; Ravel, J. An improved dual-indexing approach for multiplexed 16S rRNA gene sequencing on the Illumina MiSeq platform. Microbiome 2014, 2, 6. [CrossRef] [PubMed]

36. Igolkina, A.A.; Grekhov, G.A.; Pershina, E.V.; Samosorova, G.G.; Leunova, V.M.; Semenova, A.N.; Baturina, O.A.; Kabilov, M.R.; Andronov, E.E. Identifying components of mixed and contaminated soil samples by detecting specific signatures of control $16 \mathrm{~S}$ rRNA libraries. Ecol. Indic. 2018, 94, 446-453. [CrossRef]

37. Edgar, R.C. UPARSE: Highly accurate OTU sequences from microbial amplicon reads. Nat. Methods 2013, 10, 996-998. [CrossRef]

38. Edgar, R.C. SINTAX: A Simple Non-Bayesian Taxonomy Classifier for 16S and ITS Sequences. bioRxiv 2016. [CrossRef]

39. Wang, Q.; Garrity, G.M.; Tiedje, J.M.; Cole, J.R. Naïve Bayesian Classifier for Rapid Assignment of rRNA Sequences into the New Bacterial Taxonomy. Appl. Environ. Microbiol. 2007, 73, 5261-5267. [CrossRef]

40. Fauth, E.; Bernardo, J.; Camara, M.; Resetarits, W.J.; van Buskirk, J.; McCollum, S.A. Simplifying the Jargon of Community Ecology: A Conceptual Approach. Am. Naturalist. 1996, 147, 282-286. [CrossRef]

41. Ahmad, A.; Yang, W.; Chen, G.; Shafiq, M.; Javed, S.; Ali Zaidi, S.S.; Shahid, R.; Liu, C.; Bokhari, H. Analysis of gut microbiota of obese individuals with type 2 diabetes and healthy individuals. PLoS ONE 2019, 14, e0226372. [CrossRef] [PubMed]

42. Fujio-Vejar, S.; Vasquez, Y.; Morales, P.; Magne, F.; Vera-Wolf, P.; Ugalde, J.A.; Navarrete, P.; Gotteland, M. The Gut Microbiota of Healthy Chilean Subjects Reveals a High Abundance of the Phylum Verrucomicrobia. Front. Microbiol. 2017, 8, 1221. [CrossRef] [PubMed]

43. Ozato, N.; Saito, S.; Yamaguchi, T.; Katashima, M.; Tokuda, I.; Sawada, K.; Katsuragi, Y.; Kakuta, M.; Imoto, S.; Ihara, K.; et al. Blautia genus associated with visceral fat accumulation in adults 20-76 years of age. NPJ Biofilms Microbiomes 2019, 5, 28. [CrossRef] [PubMed]

44. Vaiserman, A.; Romanenko, M.; Piven, L.; Moseiko, V.; Lushchak, O.; Kryzhanovska, N.; Guryanov, V.; Koliada, A. Differences in the gut Firmicutes to Bacteroidetes ratio across age groups in healthy Ukrainian population. BMC Microbiol. 2020, $20,221$. [CrossRef]

45. Janulewicz, P.A.; Seth, R.K.; Carlson, J.M.; Ajama, J.; Quinn, E.; Heeren, T.; Klimas, N.; Lasley, S.M.; Horner, R.D.; Sullivan, K.; et al. The Gut-Microbiome in Gulf War Veterans: A Preliminary Report. Int. J. Environ. Res. Publ. Health 2019, 16, 3751. [CrossRef]

46. Ren, Z.; Fan, Y.; Li, A.; Shen, Q.; Wu, J.; Ren, L.; Lu, H.; Ding, S.; Ren, H.; Liu, C.; et al. Alterations of the Human Gut Microbiome in Chronic Kidney Disease. Adv. Sci. 2020, 7, 2001936. [CrossRef]

47. Walker, A.W.; Ince, J.; Duncan, S.H.; Webster, L.M.; Holtrop, G.; Ze, X.; Brown, D.; Stares, M.D.; Scott, P.; Bergerat, A.; et al. Dominant and diet-responsive groups of bacteria within the human colonic microbiota. ISME J. 2011, 5, 220-230. [CrossRef]

48. Arumugam, M.; Raes, J.; Pelletier, E.; Le Paslier, D.; Yamada, T.; Mende, D.R.; Femandes, G.R.; Tap, J.; Bruls, T.; Batto, J.-M.; et al. Enterotypes of the human gut microbiome. Nature 2011, 473, 174-180. [CrossRef]

49. Kozhieva, M.; Naumova, N.; Alikina, T.; Boyko, A.; Vlassov, V.; Kabilov, M. Primary progressive multiple sclerosis in a Russian cohort: Relationship with gut bacterial diversity. BMC Microbiol. 2019, 19, 309. [CrossRef]

50. Valeriani, F.; Gallè, F.; Cattaruzza, M.S.; Antinozzi, M.; Gianfranceschi, G.; Postiglione, N.; Romano Spica, V.; Liguori, G. Are nutrition and physical activity associated with gut microbiota? A pilot study on a sample of healthy young adult. Ann. Ig. 2020, 32, 521-527. [CrossRef] 
51. Rinninella, E.; Cintoni, M.; Raoul, P.; Lopetuso, L.R.; Scaldaferri, F.; Pulcini, G.; Miggiano, G.A.D.; Gasbarrini, A.; Mele, M.C. Food Components and Dietary Habits: Keys for a Healthy Gut Microbiota Composition. Nutrients 2019, 11, 2393. [CrossRef] [PubMed]

52. Odamaki, T.; Kato, K.; Sugahara, H.; Hashikura, N.; Takahashi, S.; Xiao, J.Z.; Abe, F.; Osawa, R. Age-related changes in gut microbiota composition from newborn to centenarian: A cross-sectional study. BMC Microbiol. 2016, 16, 90. [CrossRef] [PubMed]

53. Radjabzadeh, D.; Boer, C.G.; Beth, S.A.; van der Wal, P.; Kiefte-De Jong, J.C.; Jansen, M.; Konstantinov, S.R.; Peppelenbosch, M.P.; Hays, J.P.; Jaddoe, V.; et al. Diversity, compositional and functional differences between gut microbiota of children and adults. Sci. Rep. 2020, 10, 1040. [CrossRef] [PubMed]

54. Loman, B.R.; Shrestha, C.L.; Thompson, R.; Groner, J.A.; Mejias, A.; Ruoff, K.L.; O’Toole, G.A.; Bailey, M.T.; Kopp, B.T. Age and environmental exposures influence the fecal bacteriome of young children with cystic fibrosis. Pediatric Pulmonol. 2020, 55, 1661-1670. [CrossRef]

55. Dominianni, C.; Sinha, R.; Goedert, J.J.; Pei, Z.; Yang, L.; Hayes, R.B.; Ahn, J. Sex, body mass index, and dietary fiber intake influence the human gut microbiome. PLoS ONE 2015, 10, e0124599. [CrossRef]

56. Gallè, F.; Valeriani, F.; Cattaruzza, M.S.; Gianfranceschi, G.; Liguori, R.; Antinozzi, M.; Mederer, B.; Liguori, G.; Romano Spica, V. Mediterranean Diet, Physical Activity and Gut Microbiome Composition: A Cross-Sectional Study among Healthy Young Italian Adults. Nutrients 2020, 12, 2164. [CrossRef]

57. Egshatyan, L.; Kashtanova, D.; Popenko, A.; Tkacheva, O.; Tyakht, A.; Alexeev, D.; Karamnova, N.; Kostryukova, E.; Babenko, V.; Vakhitova, M.; et al. Gut microbiota and diet in patients with different glucose tolerance. Endocr. Connect. 2016, 5, 1-9. [CrossRef]

58. Le Chatelier, E.; Nielsen, T.; Qin, J.; Prifti, E.; Hildebrand, F.; Falony, G.; Almeida, M.; Arumugam, M.; Batto, J.M.; Kennedy, S.; et al. Richness of human gut microbiome correlates with metabolic markers. Nature 2013, 500, 541-546. [CrossRef]

59. Markle, J.G.; Frank, D.N.; Mortin-Toth, S.; Robertson, C.E.; Feazel, L.M.; Rolle-Kampczyk, U.; von Bergen, M.; McCoy, K.D.; Macpherson, A.J.; Danska, J.S. Sex differences in the gut microbiome drive hormone-dependent regulation of autoimmunity. Science 2013, 339, 1084-1088. [CrossRef]

60. Katz Sand, I.; Zhu, Y.; Ntranos, A.; Clemente, J.C.; Cekanaviciute, E.; Brandstadter, R.; Crabtree-Hartman, E.; Singh, S.; Bencosme, Y.; Debelius, J.; et al. Disease-modifying therapies alter gut microbial composition in MS. Neurol. Neuroimmunol. Neuroinflamm. 2019, 6, e517. [CrossRef]

61. Castillo-Álvarez, F.; Pérez-Matute, P.; Oteo, J.A.; Marzo-Sola, M.E. The influence of interferon $\beta-1 b$ on gut microbiota composition in patients with multiple sclerosis. Neurologia 2018. [CrossRef]

62. Storm-Larsen, C.; Myhr, K.M.; Farbu, E.; Midgard, R.; Nyquist, K.; Broch, L.; Berg-Hansen, P.; Buness, A.; Holm, K.; Ueland, T.; et al. Gut microbiota composition during a 12-week intervention with delayed-release dimethyl fumarate in multiple sclerosis-A pilot trial. Mult. Scler. J. Exp. Transl. Clin. 2019, 5. [CrossRef] [PubMed]

63. Jain, A.; Li, X.H.; Chen, W.N. Similarities and differences in gut microbiome composition correlate with dietary patterns of Indian and Chinese adults. AMB Express 2018, 8, 104. [CrossRef] [PubMed]

64. Santos-Marcos, J.A.; Haro, C.; Vega-Rojas, A.; Alcala-Diaz, J.F.; Molina-Abril, H.; Leon-Acuña, A.; Lopez-Moreno, J.; Landa, B.B.; Tena-Sempere, M.; Perez-Martinez, P.; et al. Sex Differences in the Gut Microbiota as Potential Determinants of Gender Predisposition to Disease. Mol. Nutr. Food Res. 2019, 63, e1800870. [CrossRef] [PubMed]

65. Hiippala, K.; Jouhten, H.; Ronkainen, A.; Hartikainen, A.; Kainulainen, V.; Jalanka, J.; Satokari, R. The Potential of Gut Commensals in Reinforcing Intestinal Barrier Function and Alleviating Inflammation. Nutrients 2018, 10, 988. [CrossRef]

66. Cignarella, F.; Cantoni, C.; Ghezzi, L.; Salter, A.; Dorsett, Y.; Chen, L.; Phillips, D.; Weinstock, G.M.; Fontana, L.; Cross, A.H.; et al. Intermittent Fasting Confers Protection in CNS Autoimmunity by Altering the Gut Microbiota. Cell Metab. 2018, 27, 1222-1235.e6. [CrossRef] 